\title{
Localization of triplet excitons and biexcitons in the two-dimensional semiconductor $\left(\mathrm{CH}_{3} \mathrm{C}_{6} \mathrm{H}_{4} \mathrm{CH}_{2} \mathrm{NH}_{3}\right)_{2} \mathrm{PbBr}_{4}$
}

\author{
T. Goto, H. Makino,* and T. Yao \\ Center for Interdisciplinary Research, Tohoku University, Sendai 980-8578, Japan \\ C. H. Chia, T. Makino, and Y. Segawa \\ Photodynamics Research Center, RIKEN (Institute of Physical and Chemical Research), Sendai 980-0845, Japan
}

G. A. Mousdis and G. C. Papavassiliou

Theoretical and Physical Chemistry Institute, National Hellenic Research Foundation, Athens 116-35, Greece

(Received 3 July 2005; revised manuscript received 29 November 2005; published 21 March 2006)

\begin{abstract}
We have measured emission spectra under selective excitation and time resolved photoluminescence spectra of a single crystal with a perovskite type structure $\left(\mathrm{CH}_{3} \mathrm{C}_{6} \mathrm{H}_{4} \mathrm{CH}_{2} \mathrm{NH}_{3}\right)_{2} \mathrm{PbBr}_{4}$ at low temperature, and clarified that triplet excitons generated in the $\mathrm{PbBr}$ monolayer are recombined after localization in a random potential. The localized triplet exciton has a lifetime below $2.3 \mathrm{~ns}$. From emission spectra under selective excitation of a strong nanosecond pulsed light, it is suggested that free and localized biexcitons are generated by inelastic collision of two free and localized triplet excitons, respectively. Moreover, it is concluded that the localized biexciton is annihilated radiatively leaving a localized triplet exciton.
\end{abstract}

DOI: 10.1103/PhysRevB.73.115206

PACS number(s): 71.35.-y, 78.67.De

\section{INTRODUCTION}

Optical properties of perovskite type materials composed of organic and inorganic materials have been extensively studied because of electronic dimensionality, extremely large exciton binding energy, and large optical nonlinearities. Another characteristics is that crystals with zero-, one-, two-, and three-dimensional structures exist. ${ }^{1,2}$ Among them, twodimensional crystals have a superlattice structure with an inorganic well monolayer and an organic barrier layer. In the absorption spectrum of the thin film, structures due to a dipole allowed singlet exciton $(X)$ in the inorganic monolayer are observed. A quantum well composed of a lead bromidebased monolayer has a larger exciton binding energy $i>430 \mathrm{meV}$ (Ref. 2) $j$ than that of the more common iodidebased monolayer, ${ }^{3,4}$ and hence, the larger optical nonlinearity is expected. Recently, we observed clear and sharp structures due to the $X$ in the reflection and photoluminescence (PL) spectra, and due to a dipole-forbidden triplet exciton (TX) in the absorption and PL spectra using a single crystal with excellent quality. ${ }^{5}$

On the other hand, a biexciton, i.e., an excitonic molecule $(X X)$ is very important for nonlinear optical devices, because the $X X$ is an intermediate state of nonlinear optical processes. In lead-iodide based crystals, the $X X$ with a binding energy, circa $40 \mathrm{meV}$, has been found by Kondo et al. ${ }^{6}$ Such a large binding energy is caused by the extremely large binding energy, circa $310 \mathrm{meV}$ of the $X{ }^{4}$ Kato et al. have reported the largest binding energy of the $X X$ to be $60 \mathrm{meV}$ in $\left(\mathrm{C}_{4} \mathrm{H}_{9} \mathrm{NH}_{3}\right)_{2} \mathrm{PbBr}_{4}$ (Ref. 7). In the previous paper, ${ }^{5}$ we reported the $X X$ with a binding energy of $38 \mathrm{meV}$ in $\left(\mathrm{CH}_{3} \mathrm{C}_{6} \mathrm{H}_{4} \mathrm{CH}_{2} \mathrm{NH}_{3}\right)_{2} \mathrm{PbBr}_{4}$, which is smaller than that reported by Kato et al. This difference in the biexciton binding energy is originated from an assignment that $X X$ is formed not from $X$ but from $T X .^{5}$
On the other hand, localization of excitons ${ }^{8}$ and biexcitons ${ }^{9}$ in mixed crystals, and of excitons ${ }^{10-12}$ and biexcitons ${ }^{13}$ in quantum well structures have been found. However, their localization has not been reported in perovskite-type structures composed of organic and inorganic materials, as far as we know.

In the present paper, localization of both $T X$ and $X X$ is clarified by analyses of absorption spectra, emission spectra under selective excitations, and time resolved PL spectra. Localization of $T X$ and $X X$ is characteristic of the quasi-twodimensional motion. Moreover, it is concluded that the localized $X X$ is generated by the inelastic collision of two localized $T X$ 's as well as by relaxation from the free $X X$.

\section{EXPERIMENTAL DETAILS}

A single crystal of $\left(\mathrm{CH}_{3} \mathrm{C}_{6} \mathrm{H}_{4} \mathrm{CH}_{2} \mathrm{NH}_{3}\right)_{2} \mathrm{PbBr}_{4}$ was prepared by treating $\mathrm{PbO}$ and $\mathrm{CH}_{3} \mathrm{C}_{6} \mathrm{H}_{4} \mathrm{CH}_{2} \mathrm{NH}_{3} \mathrm{Br}$ with $\mathrm{HBr}$ in

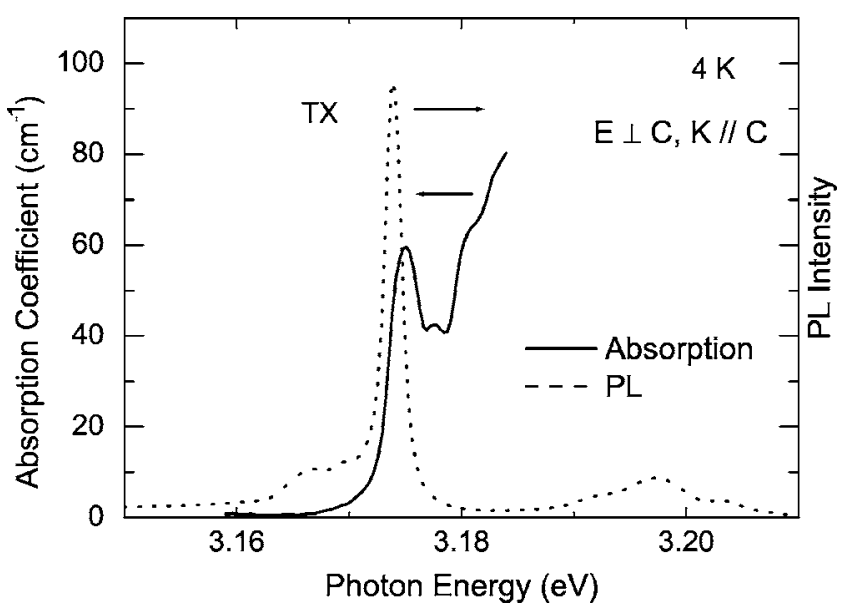

FIG. 1. Absorption and PL spectra at $4 \mathrm{~K}$ for orientation $\mathbf{E} \perp \mathbf{C}$, $\mathbf{K} \| \mathbf{C}$ are shown by solid and dashed lines, respectively. 


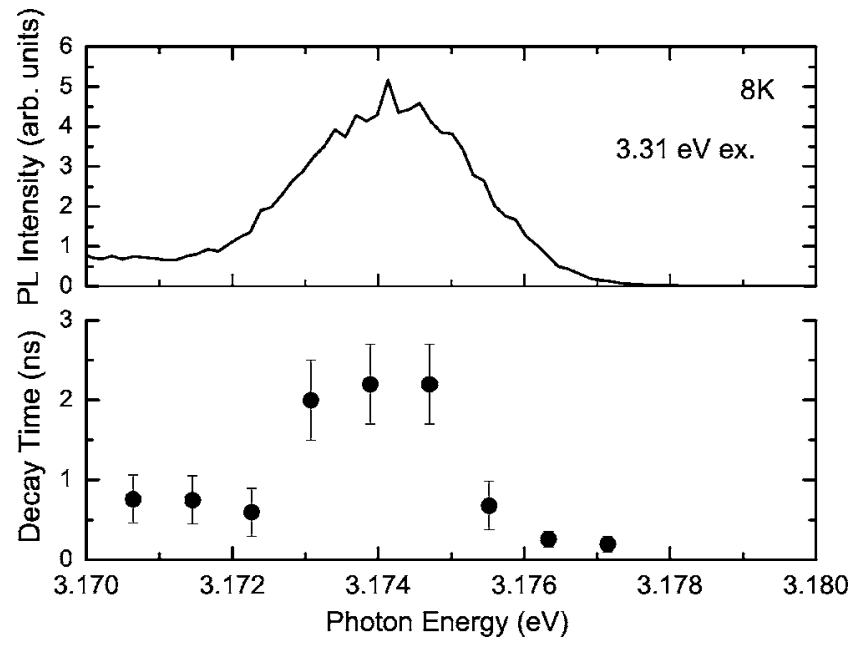

FIG. 2. The PL intensity and decay time as a function of the photon energy around the $T X$ lines at $8 \mathrm{~K}$ are shown in the upper and lower panels, respectively. The excitation photon energy is $3.31 \mathrm{eV}$, and the spectral resolution is $1 \mathrm{meV}$.

$\mathrm{CH}_{3} \mathrm{OH}$. The preparation details have been reported by $\mathrm{Pa}-$ pavassiliou et al. ${ }^{14}$ It has a $\mathrm{P} 2$ / $a$ crystal structure, and consists of alternating well layers, i.e., two-dimensional networks of $\mathrm{PbBr}_{6}$ octahedrons, and barrier layers of 4-methylbenzylamine $\left(\mathrm{CH}_{3} \mathrm{C}_{6} \mathrm{H}_{4} \mathrm{CH}_{2} \mathrm{NH}_{3}\right)$. The distance between the well layers is about $1.8 \mathrm{~nm}$ and the distance between the octahedrons is $0.8 \mathrm{~nm}$. The crystal used in this work was platelet shaped and the dimension was approximately $3 \times 3 \times 0.025 \mathrm{~mm}^{3}$.

We measured reflection and emission spectra by the following method. The crystal was attached to a copper sample holder of a cryogenic refrigerator with silver paste and the sample holder was cooled down to $4 \mathrm{~K}$. For measuring the transmission spectrum, a deuterium lamp was used as an incident light source, and the transmitted light was analyzed by a monochromator with reciprocal dispersion, $14 \mathrm{meV} / \mathrm{mm}$, and detected by a silicon photodiode array. The spectral resolution was $0.7 \mathrm{meV}$. In usual PL measurements, the crystal was excited by $3.81 \mathrm{eV}$ photons from a $6 \mathrm{~mW} \mathrm{He}-\mathrm{Cd}$ laser. The emission was analyzed and detected by the identical aforementioned setup. The polarized light was obtained using a polar film, and the temperature was measured with a silicon diode temperature sensor. In the time-resolved PL measurements, the following excitation and detection systems were used; the exciting light was supplied using an argon laser, a mode-locked Ti:sapphire laser and a second harmonic generation unit, with $3.31 \mathrm{eV}$ photons, a repetition rate of $82 \mathrm{MHz}$, and a pulse width of $2 \mathrm{ps}$. The emission detection system was composed of a monochromator with a reciprocal dispersion of $23 \mathrm{meV} / \mathrm{mm}$ and a Hamamatsu streak camera. The spectral resolution was $1 \mathrm{meV}$, and the time resolution was $20 \mathrm{ps}$.

For measurements of emissions under high excitation power, the third harmonic of a $Q$-switched Nd-YAG laser with $3.49 \mathrm{eV}$ photons, a pulse width of $5 \mathrm{~ns}$, and a repetition rate less than $10 \mathrm{~Hz}$ was used as an exciting light source. The maximum intensity on the crystal surface was approximately $100 \mathrm{~kW} / \mathrm{cm}^{2}$. For the emission measurements under high

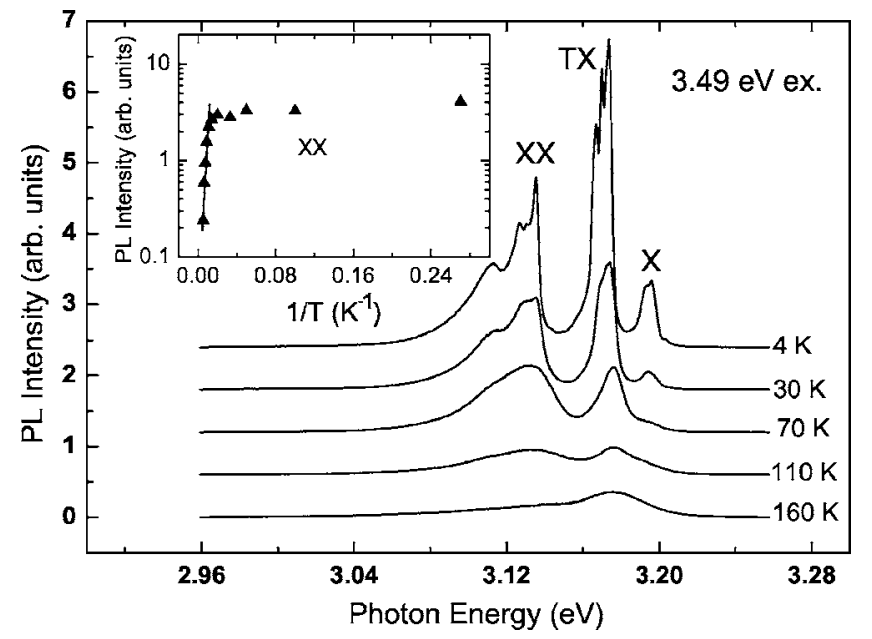

FIG. 3. PL spectra at different temperatures under excitation with $3.49 \mathrm{eV}$ photons. The inset shows the temperature dependence of the $X X$ intensity. A straight line represents thermal activation energy of $36 \mathrm{meV}$.

density and selective excitations, we used the second harmonic of a tunable infrared light from a dye laser pumped by the second harmonic of a YAG laser. The pulse width and the repetition rate were $20 \mathrm{~ns}$ and $50 \mathrm{~Hz}$, respectively. The maximum intensity on the sample surface was about $160 \mathrm{~kW} / \mathrm{cm}^{2}$. The emission from the sample was analyzed by a double monochromator with a reciprocal dispersion of $5 \mathrm{meV} / \mathrm{mm}$ and detected by a charge coupled device camera. The spectral resolution was better than $0.8 \mathrm{meV}$.

\section{EXPERIMENTAL RESULTS AND DISCUSSION}

\section{A. Excitons}

Figure 1 shows absorption (solid line) and PL (dashed line) spectra at $4 \mathrm{~K}$ for an orientation: $\mathbf{E} \perp \mathbf{C}, \mathbf{K} \| \mathbf{C}$, where $\mathbf{K}, \mathbf{E}$, and $\mathbf{C}$ represent the light propagation vector, the light electric vector, and the $c$ axis of the crystal, respectively. A very narrow absorption line with the full width at half maximum (FWHM) of $2 \mathrm{meV}$ appears at $3.175 \mathrm{eV}$ whose absorption coefficient is four orders of magnitude smaller than that of the direct allowed exciton absorption lines. ${ }^{5}$ From the very small oscillator strength, it is suggested that this line is associated with $T X$. The small oscillator strength may be caused by mixing of the $T X$ wave function with a singlet one through a spin-orbit interaction. In the PL spectrum, a strong and narrow line appears at $3.174 \mathrm{eV}$, which is located $1 \mathrm{meV}$ to the lower energy side compared to the main $T X$ absorption line. This PL line has been assigned as the $T X$ in the previous paper. ${ }^{5}$ The origin of the Stokes shift will be discussed later. In addition to this line, there appear a few weak lines on the higher energy side of the $3.175 \mathrm{eV}$ absorption line and on the lower energy side of the $3.174 \mathrm{eV}$ PL line. These lines are associated with $T X$ 's in different polytypes, like the case of the direct-allowed excitons, ${ }^{5}$ the PL lines of which are observed around $3.198 \mathrm{eV}$.

The PL intensities of the $T X$ lines decrease exponentially with time after the pulsed excitation, not shown here. The 


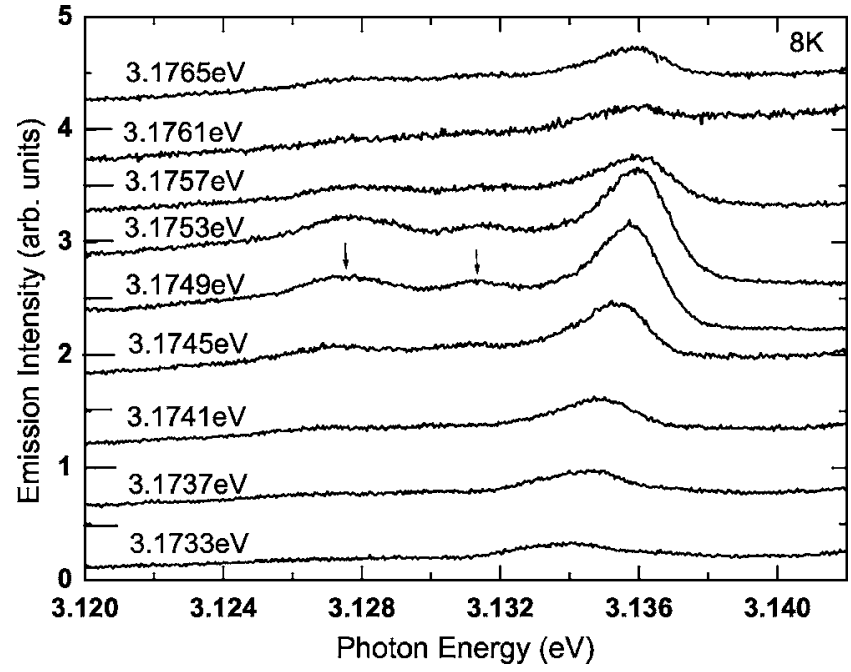

FIG. 4. Emission spectra near the $X X$ line at different excitation energies around the main $T X$ absorption line at $8 \mathrm{~K}$. The excitation intensity is about $160 \mathrm{~kW} / \mathrm{cm}^{2}$.

time-integrated PL intensity and the decay time of the $T X$ lines are shown in the top and bottom panels, respectively, of Fig. 2 as a function of photon energy at $8 \mathrm{~K}$. The energy of the excitation light, $3.31 \mathrm{eV}$, corresponds to the higher energy tail of the $X$ absorption band. The abscissa is expanded in comparison with that of Fig. 1. The decay time is $0.2 \mathrm{~ns}$ at the higher energy edge of the $T X$ line, and is $2.3 \mathrm{~ns}$ at $3.1747 \mathrm{eV}$. Below $3.1723 \mathrm{eV}$, the decay time drops again down to $\sim 0.8 \mathrm{~ns}$. In this energy region, however, a weak line due to the $T X$ in a different polytype is located, as seen in the PL spectrum of Fig. 1. Hence, the short decay times below $3.1723 \mathrm{eV}$ may be caused by the PL in the different polytype. Thus, this dependence means that the lifetime of the $T X$ is longer than that of the $X$. From this fact, it is suggested that the $T X$ is localized in a random potential, which results in the $T X$ dropping toward a lower energy level with a longer lifetime than that of a free $T X$. So, the observed 0.2 ns lifetime at the higher energy side of the main PL peak can be attributed to a free $T X$, while the longer lifetimes are due to localized $T X$ 's. The redshift of the PL line compared to the absorption line as seen in Fig. 1 is attributed to the phonon relaxation from the free $T X$ to the localized $T X$, as reabsorption of the PL is rarely observed. Namely, the main absorption line in Fig. 1 is associated with the free $T X$, and the main PL line is associated with the localized $T X$. As the spectral resolution of $1 \mathrm{meV}$ is not fine enough to measure exactly the absorption and PL line shapes, decay times longer than $0.2 \mathrm{~ns}$ are obtained even near the $T X$ absorption line peak of $3.175 \mathrm{eV}$. The existence of the localized $T X$ is also suggested from direct generation of the localized $X X$ as stated later. The random potential may be caused by disorder of the atomic arrangement in the boundaries between the well monolayers composed of $\mathrm{PbBr}_{6}$ octahedrons and the organic barrier layers. Similar exciton localization at interface boundaries have been reported in semiconductor quantum wells such as GaAs/AlAs, ${ }^{10} \mathrm{GaAs} / \mathrm{AlGaAs},{ }^{11}$ and $\mathrm{ZnO} / \mathrm{ZnMgO}$ (Ref. 12) systems.

On the other hand, the decay time of the $X$-PL was measured to be less than 20 ps. Hence, the lifetime of the free $T X$

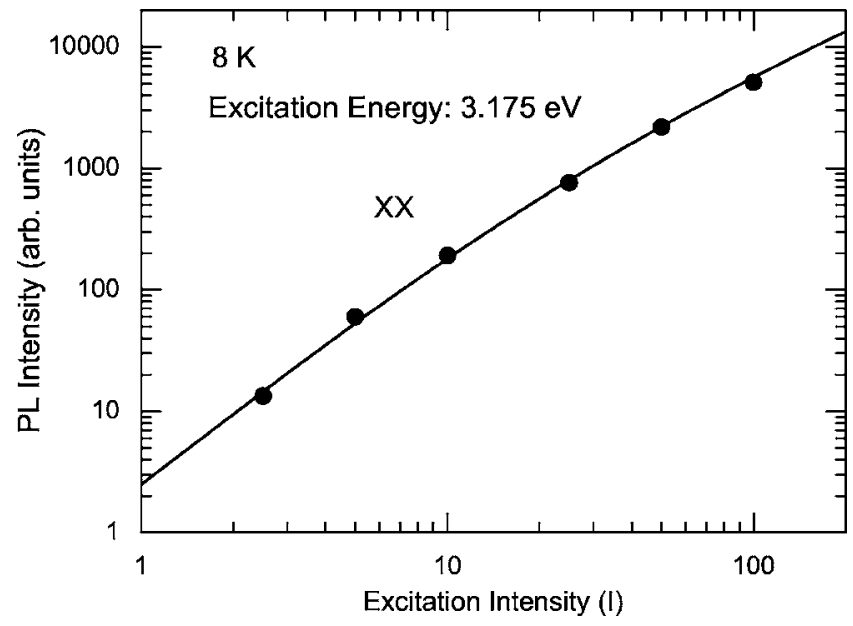

FIG. 5. PL intensity of the $X X$ line vs excitation intensity $(I)$ at $8 \mathrm{~K}$ on a logarithmic scale. The excitation energy is $3.175 \mathrm{eV}$. Closed circles show experimental points. A solid line represents Eq. (1) with an adjustable parameter $I_{0}=10$.

is much longer than that of the $X$. As the $T X$ state is mixed with the singlet state through spin-orbit interactions, finite absorption and finite lifetime are observed.

\section{B. Biexcitons}

Next, we report on optical phenomena and mechanisms under excitations by strong pulsed lights. Figure 3 shows the PL spectra at different temperatures under the $3.49 \mathrm{eV}$ photon excitation with a power of about $100 \mathrm{~kW} / \mathrm{cm}^{2}$. The temperature of the $T X$ system is raised up to $14 \mathrm{~K}$ by the strong pulsed light irradiation, when the holder temperature is $4 \mathrm{~K}$. The temperature of the $T X$ system is estimated from the blueshift of the $T X$ absorption peak during the pulsed light irradiation. In the spectrum at $4 \mathrm{~K}$, lines at $3.195 \mathrm{eV}, 3.174 \mathrm{eV}$, and $3.136 \mathrm{eV}$, have been assigned as due to $X, T X$, and $X X$, respectively. ${ }^{5}$ The temperature dependence of the $X X$ line intensity is plotted by triangles as a function of the inverse temperature $1 / T$ in the inset. A straight line represents thermal activation energy of $36 \mathrm{meV}$, which is nearly equal to the optical binding energy of $38 \mathrm{meV}{ }^{5}$ With the temperature rise from 4 to $70 \mathrm{~K}$, peak energies of the $T X$ and $X X$ lines are blueshifted, which reflects the temperature shift of the band gap energy, because the $X$ absorption lines show the same shift. On the contrary, the $X$ lines seem to be redshifted. This fact may be caused by thermal relaxation of the $X$ to the lower energy levels in different polytypes.

In order to study the $X X$ formation process, we measured emission spectra when the crystal is excited by tunable pulsed light. Figure 4 shows emission spectra under excitation at different photon energies (shown in the figure) around the main $T X$ absorption line at $8 \mathrm{~K}$. The excitation light intensity is about $160 \mathrm{~kW} / \mathrm{cm}^{2}$ on the crystal surface. The excitation energy of $3.1749 \mathrm{eV}$ corresponds to the peak energy of the $T X$ absorption line. When the excitation energy is equal to the peak energy of the $T X$ absorption line, $3.1750 \mathrm{eV}$, the intensity of the $X X$ line near $3.136 \mathrm{eV}$ reaches a maximum. Two lines shown by arrows at 3.1275 


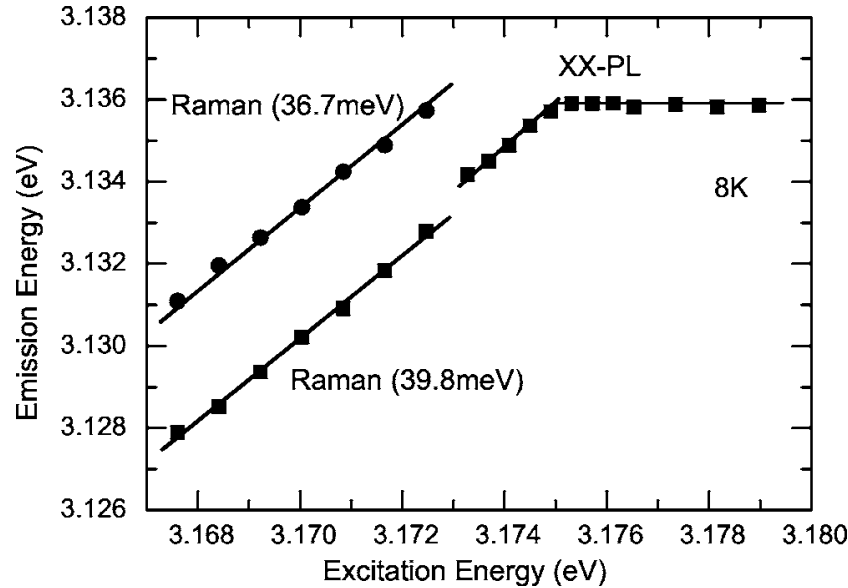

FIG. 6. Closed circles and squares show the relation between the emission and excitation energies. Oblique and horizontal straight lines represent a linear emission energy dependence of the excitation energy and a constant relation of the emission energy to the excitation energy, respectively.

and $3.1315 \mathrm{eV}$ are associated with phonon replicas of the $X X$ line..$^{5}$

The PL intensity of the $X X$ line as a function of the excitation intensity, $I$, is shown by closed circles in Fig. 5, where the excitation photon energy equals the peak energy of the $T X$ absorption line. A relation between the $X X$ density, $n$, and the $I$, in a system of coexisting excitons and biexcitons is given by

$$
n \propto\left\{\left(1+I / I_{0}\right)^{1 / 2}-1\right\}^{2},
$$

where $I_{0}$ is the intensity characteristic of an intermediate state between the $X$ and $X X$ dominant regions. ${ }^{15}$ Here, the $X$ and $X X$ are formed by a photon and two $X \mathrm{~s}$, respectively, and the $X X$ is annihilated radiatively leaving an $X$. When the PL intensity is proportional to the $n$, Eq. (1) with an adjustable parameter $I_{0}=10$ is represented by a solid line in Fig. 5 . The calculated curve is in good agreement with the experimental points. This means that the $X X$ is directly generated from a collision of two $T X$ 's.

The circles and squares of Fig. 6 show the relation between the emission and excitation energies. Based on the constant relation of the $X X$ emission to the excitation photon energy above $3.175 \mathrm{eV}$, it is suggested that a free $T X$ generated by light is relaxed near the lowest energy level through phonon scattering and then the free $X X$ is formed by the collision of the two thermalized $T X$ 's, as shown on the left side of Fig. 7. Probably, the free $X X$ becomes localized $X X$, and then emits a photon leaving a localized $T X$ behind.

Of particular note is the linear relation of the $X X$-PL energy to the excitation energy in the energy range less than $3.175 \mathrm{eV}$, which is the peak energy of the $T X$ absorption line. From this linear relation, we propose the following mechanism. A localized $T X$ is directly generated by a photon, as shown on the right side of Fig. 7. The two localized $T X$ 's interact with each other, which results in a localized $X X$, because even the localized $T X$ is able to hop in a random potential. The localized $X X$ recombines radiatively leaving a
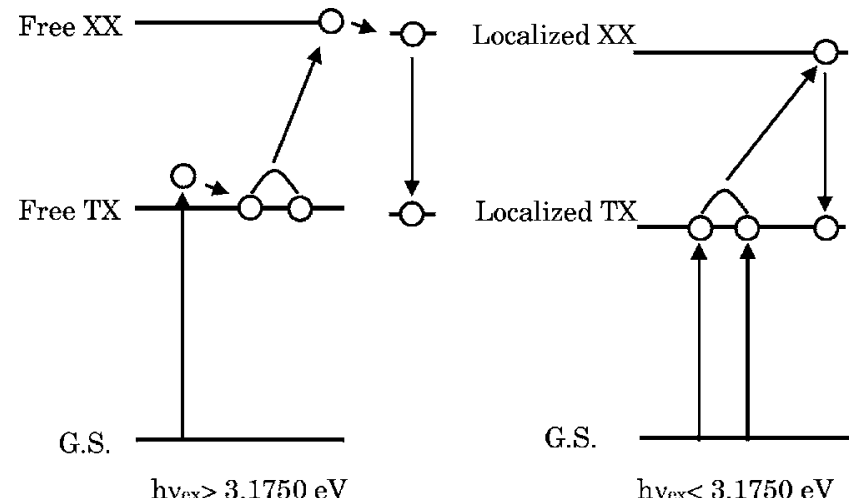

$h v_{\mathrm{ex}}>3.1750 \mathrm{eV}$

$h v_{\text {ex }}<3.1750 \mathrm{eV}$

FIG. 7. Energy diagram of the $T X$ and $X X$. Proposed optical processes under excitations of photons with energies above and below $3.175 \mathrm{eV}$ are shown on the left and right sides, respectively. GS means the ground state. Optical transitions are shown by vertical arrows. Horizontal lines for the free $T X$ and $X X$ represent the lowest energy level.

localized $T X$. In this process, the energy relation between the emitting $\left(h \nu_{\mathrm{e}}\right)$ and exciting $\left(h \nu_{\mathrm{ex}}\right)$ photons is written as

$$
\begin{aligned}
h \nu_{\mathrm{e}} & =E_{L X X}-E_{L T X}=\left(2 E_{L T X}-E_{L X X}^{B}\right)-E_{L T X} \\
& =E_{L T X}-E_{L X X}^{B}=h \nu_{\mathrm{ex}}-E_{L X X}^{B},
\end{aligned}
$$

where $E_{L X X}$ and $E_{L T X}$ are formation energies of the localized $X X$ and $T X$, respectively, and $E_{L X X}^{B}$ the binding energy of the localized $X X$. By making a comparison between Eq. (2) and Fig. 6, $E_{L X X}^{B}$ is obtained as $39 \mathrm{meV}$, the value of which is very close to the previously reported value, $38 \mathrm{meV}$, of the $X X{ }^{5}$ The $X X$ line disappears under the excitation energies below $3.1723 \mathrm{eV}$, which corresponds to the lower energy edge of the main $T X$ absorption line and to the lower excitation energy edge showing the long decay time in Fig. 2. This fact means that the $X X$ cannot be generated by light with the energy below $3.1723 \mathrm{eV}$ but the Raman scattering occurs instead.

The PL excitation (PLE) spectrum of the $X X$ line is shown by circles in Fig. 8 and compared with the absorption spec-

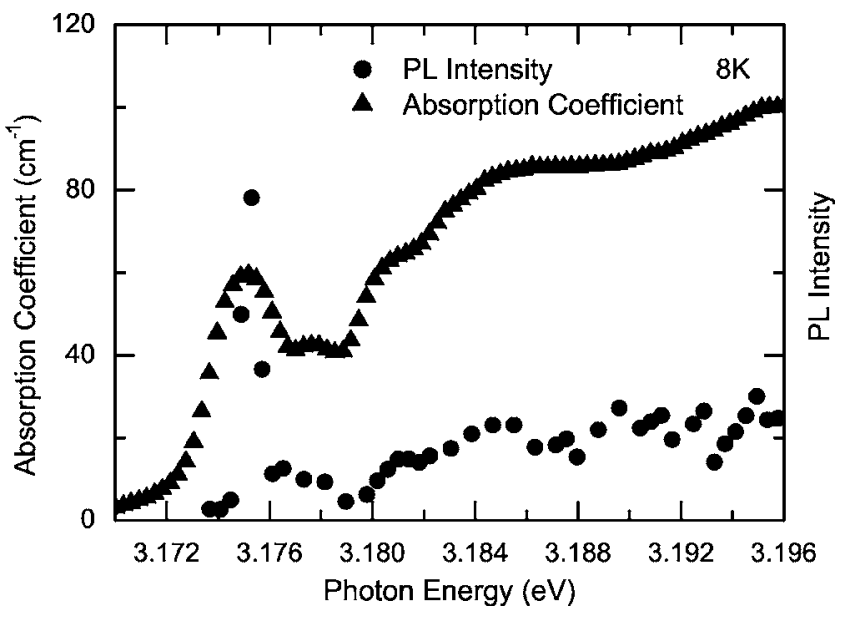

FIG. 8. A PLE spectrum for the $X X$ and the absorption spectrum around the $T X$ at $8 \mathrm{~K}$ is shown by circles and triangles, respectively. 
trum shown by triangles. The main peak energy in the PLE spectrum coincides with that in the absorption spectrum. Energies of a few peaks on the higher energy side in the PLE spectrum are also the same as those in the absorption spectrum. From this close correspondence, the direct $X X$ formation from the $T X$ is strongly supported.

\section{SUMMARY}

In summary, from the finite Stokes shift of the $T X$-PL line and the excitation energy dependence of the decay time it is concluded that the $T X$ is annihilated after localization at a random potential field. Moreover, we have found a coincidence between the optical and thermal binding energies of the $X X$. Next, we measured emission spectra at selective excitations around the $T X$ energy using strong nanosecond pulsed light. It is concluded from the experimental results that the $X X$ is also localized and then annihilates radiatively leaving a localized $X X$. Free and localized $X X$ 's are generated by inelastic collision of two free and two localized $T X$ 's, respectively. The localized $X X$ is annihilated radiatively leaving a localized $T X$.

\section{ACKNOWLEDGMENTS}

This work was partially supported by the Ministry of Education, Culture, Sports, Science and Technology of Japan through a Grant-in-Aid for Young Scientists (B) (Grant No. 16760005).
*Present affiliation: Research Institute, Kochi University of Technology, Tosayamada, Kochi 782-8502, Japan.

${ }^{1}$ T. Ishihara, Optical Properties of Low Dimensional Materials, edited by T. Ogawa and Y. Kanemitsu (World Scientific, Singapore, 1995), Vol. 1, p. 288.

${ }^{2}$ G. C. Papavassiliou, Prog. Solid State Chem. 25, 125 (1997).

${ }^{3}$ T. Goto, N. Ohshima, G. A. Mousdis, and G. C. Papavassiliou, Solid State Commun. 117, 13 (2001)

${ }^{4}$ K. Tanaka, F. Sano, T. Takahashi, T. Kondo, R. Ito, and K. Era, Solid State Commun. 122, 249 (2002)

${ }^{5}$ H. Makino, T. Goto, T. Yao, G. A. Mousdis, and G. C. Papavassiliou, J. Lumin. 112, 54 (2005).

${ }^{6}$ T. Kondo, T. Azuma, T. Yuasa, and R. Ito, Solid State Commun. 105, 253 (1998)

${ }^{7}$ Y. Kato, D. Ishii, K. Ohashi, H. Kunugita, K. Ema, K. Tanaka, T. Takahashi, and T. Kondo, Solid State Commun. 128, 15 (2003).

${ }^{8}$ A. Reznitsky, S. Permogorov, S. Verbin, A. Naumov, Yu. Korostelin, Y. Novezhilov, and S. Prokovev, Solid State Commun. 52, 13 (1984); J. Takeela, T. Tayu, S. Saito, S. Kurita, T. kuga, and
M. Matsuoka, J. Lumin. 53, 507 (1992).

${ }^{9}$ T. Makino, M. Watanabe, and T. Hayashi, J. Phys. Soc. Jpn. 65, 3049 (1996).

${ }^{10}$ K. Fujiwara, H. Katahama, K. Kanamoto, R. Cingolani, and K. Ploog, Phys. Rev. B 43, 13978 (1991).

${ }^{11}$ Y. Takahashi, S. Kano, K. Muraki, S. Fukatsu, Y. Shiraki, and R. Ito, Appl. Phys. Lett. 64, 1845 (1994).

${ }^{12}$ T. Makino, K. Tamura, C. H. Chia, Y. Segawa, M. Kawasaki, A. Ohtomo, and H. Koinura, J. Appl. Phys. 93, 5929 (2003).

${ }^{13}$ U. Woggon, K. Hild, F. Gindele, W. Langbein, M. Hetterich, M. Grün, and C. Klingshirn, Phys. Rev. B 61, 12632 (2000); C. Rive, K. Varga, V. A. Schweigert, and F. M. Peeteri, Phys. Status Solidi B 210, 689 (1998).

${ }^{14}$ G. C. Papavassiliou, G. C. Mousdis, C. P. Raptopoulou, and A. Terzis, Z. Naturforsch. 55B, 536 (2000).

${ }^{15}$ P. L. Gourley and J. P. Wolfe, Phys. Rev. B 20, 3319 (1979); J. C. Kim, D. R. Wake, and J. P. Wolfe, ibid. 50, 15099 (1994); R. Spiegel, G. Bacher, A. Forchel, B. Jobst, D. Hommel, and G. Landwehr, ibid. 55, 9866 (1997). 